\title{
Telemedicine: Healthcare's Response to the COVID Crisis is Not without Disadvantages
}

\author{
Christie Kim* \\ St Joseph Health Centre, University of Toronto, Canada \\ *Corresponding author: Christie Kim, St Joseph Health Centre, University of Toronto, Canada
}

\section{ARTICLE INFO}

Received: August 03, 2020

Published: 幽 August 11, 2020

Citation: Christie Kim. Telemedicine: Healthcare's Response to the COVID Crisis is Not without Disadvantages. Biomed J Sci \& Tech Res 29(3)-2020. BJSTR. MS.ID.004806.

\author{
ABSTRACT
}

The COVID pandemic left global healthcare systems reeling. In answer to this crisis, the delivery of healthcare has rapidly incorporated telemedicine. While there arebenefits including the accessibility of care, there are significant concerns that require review. Factors affecting patient safety, including physician training, increased burden of care on patients, and ethical concerns, such as confidentiality and autonomy, may be overlooked and the quality of care may become compromised. It will be crucial to review these issues. In doing so, standards may be developed thus providing the best quality and the safest patient care.

\section{Mini Review}

Technology is rapidly reshaping the healthcare landscape. The technological innovations in medicine are improving patient care with better survival rates, shorter hospital stays and fewer complications [1,2].The development, implementation and practice of telehealth and telemedicine has been widely encouraged by administrators and used in patient care, yet as with any new medical innovation, there can be associated disadvantages. Telehealth includes the electronic telecommunications technologies, which support and promote distant patient care and professional development.Conversely, telemedicine involves an interactive virtual communication between patient and physician[3]. Although, millions of patients have received telehealth services, worldwide [1], it is interesting that telemedicine had not been readily adopted until the COVID crisis. In a 2017 report by Chaet et al., the majority of health-related technology was cloud-based imaging systems and electronic medical records, while less than $7 \%$ of patient visits were telemedicine visits [3]. Since then telemedicine has been increasingly integrated into patient care.

The COVID-19 pandemic has exponentially accelerated the use of telemedicine in most practice settings. What initially began as a regional infection in December 2019, by early 2020, the rapidly infectious coronavirus caused the worldwide COVID-19 pandemic leaving millions infected and global healthcare systems in crisis
[4]. There was an urgent need to allocate resources towards the critical care of COVID patients and prevention of further viral exposure while still maintaining quality continuity of care. This unprecedented healthcare strain accelerated the integration of telemedicine in patient management [2]. However, there are concerns that in rapidly establishing telemedicine as a new patient care model the quality of the care provided and patient safety may be compromised.

Technology has had a massive impact on healthcare with the potential to improve the quality and the safety of patient care $[5,6]$. Studies have demonstrated that telehealth has resulted in effective and timely clinical decisions, improved communication, reduced medication errors and improved patient outcomes [510]. Nonetheless, telemedicine does have disadvantages and the delivery of safe and high-quality patient care will require close review. To date studies have reported only some of the factors necessary to provide high quality telemedicine care. Aside from the obvious technological considerations, human factors including the physician, the patient and their relationship are crucial [2]. Unfortunately, as telemedicine has been rapidly incorporated into mainstream healthcare, information regarding the risks to patient safety and the quality of the care provided is either sparse or inconclusive [7]. This may be due to the lack of insight regarding 
patient safety risks with telemedicine, failure to report incidences as "safety risks" and failure to standardize reporting of telemedicine errors [6]. These issues are problematic and can interfere in the successful establishment telemedicine guidelines and standards.

Any change from standard patient care practice will inevitably be associated with an increased risk in safety.Provider education is crucial; less than $10 \%$ of clinicians report having had formal training in telemedicine, despite $55 \%$ of clinicians reporting the importance of telemedicine [11]. Thus, to mitigate these risks healthcare providers will need to undergo telemedicine training. Furthermore, the change from in-person, face-to-face care to telemedicine may hinder thorough clinical assessments and physical examinations. Systematic reviews report physicians expressing concerns regarding an increased risk in inappropriate clinical decision-making, particularly in acutely ill patients, as the patient's physical examination is limited or absent $[1,3]$.

An additional factor influencing telemedicine's success are the patients, themselves. As patients areliving longer, previously fatal diseases, such as diabetes, heart disease and some cancers, can now be managed chronically at home and as primary care moves increasingly into the community, more of the burden of care will be placed on the patients and their caregivers $[3,6,7]$. For telemedicine care to be successful, patients will be increasingly expected to work collaboratively with their providers; their communication and language proficient; their compliance with medical recommendations; and be sufficiently tech-savvy to use the remote telehealth devices or proceed with the virtual visit. However, patients in the primary care setting are increasingly older, frailer and have multiple medical issues, andtelemedicine patient care may not be feasible. This “"One Size Fits All” approach, is most appropriate for younger patients (average ages between 40-65yrs) with minimal chronic conditions and who are tech savvy [2]; a demographic vastly different from the older co-morbid primary care patients. Thus, the idea that the quality and degree of patient safety will be maintained across the spectrum of telemedicine patients must be investigated.

A key component in quality patient care involves the patientphysician relationship. This relationship requires the foundation of mutual trust and collaborative communication. Trust and communication may be jeopardized or more difficult to maintain when interactions are carried out over telemedicine instead of traditional in-person visits $[6,12,13]$. This is concerning as significant ethical issues may arise if this relationship is compromised. Without a doubt, competency, transparency, mutual trust, communication and reliability need to be maintained, regardless of the modality of care. Yet, if the patient-physician relationship changes due to telemedicine, these may be at stake. Additionally, patients will need to understand that telemedicine visits may have inherent safety risks and may not be standard of care. Thus, their autonomy will need to be considered and their informed consent will need to be explicitly stated [14,15].Another important ethical and medico-legal issue includes maintaining patient confidentiality. As data security over the internet is highly susceptible to threat and attack on patient's private information, this is of increasing concern $[9,16]$.Recognizing this issue, hospital information systems supporting telemedicine applications will need to comply with the laws regarding patient confidentiality.

While there are some disadvantages and concerns, telemedicine also has many benefits. The predominant advantageous feature is the increased accessibility of medical care, particularly, for patients in underserved or remote areas. Furthermore, patients who are homebound, have medical or psychiatric impediments that limit their access to care, or who have rare (or uncommon) medical disorders can also be cared for by physician specialists remotely $[3,17,18]$. Lastly, remote monitoring of chronic medical conditions, such as diabetes, via virtual peripheral medical devices (for example, a digital point-of-care device remotely linked to an endocrinology database) can further ensure high quality continuity of care [1618]. Having the telemedical access may also improve patient's quality of life, with less hospital visits, increased convenience and reassurance by this "virtual medical safety net" $[12,13]$.

The delivery of healthcare continues to evolve, and its landscape will include telemedicine. Undoubtedly there are numerous benefits including the accessibility of care, convenience and improved quality of life, however there are significant concerns that require close attention and review. Given the rapid rate whereby telemedicine has been integrated into patient care during this COVID crisis, factors affecting patient safety overlooked and quality of care may become compromised. It will be crucial to establish guidelines on telemedicine care, as well as standards on reporting safety risks in order to provide the highest quality of care. Having these standards would address ethical and medico-legal issues while providing guidance for physicians, medical associations and other stakeholders in establishing successful telemedicine care.

\section{References}

1. Weinstein RS, Krupinski EA, Doarn CR (2018) Clinical Examination Component of Telemedicine, Telehealth, mHealth and Connected Health Medical Practices. Med Clin N Am 102(3): 533-544.

2. Loeb AE, Rao SS, Ficke JR, Morris CD, Riley LH, et al. (2020) Departmental Experience and Lessons learned with Accelerated Introduction of Telemedicine During the CoVID-19 Crisis. Am Aca Orth Surg 28(11): e469-e476.

3. Chaet D, Clearfield R, Sabin JE, Skimming K (2107) Ethical Practice in Telehealth and Telemedicine. JGIM 32(10): 1136-1140.

4. John Hopkins COVID Resource Centre.

5. Vincent C, Amalberti R (2016) Safer Healthcare. Strategies for the Real World. Springer Open ebook.

6. McLean S, Sheikh A, Cresswell K, Nurmatov U, Mukherjee M, et al. (2013) The Impact of Telehealthcare on the Quality and Safety of Care. A Systematic Overview. PloS One 8(8): e71238. 
7. Guise V, Anderson J, Wiig S (2014) Patient Safety Risks Associated with Telecare: A Systematic Review and Narrative Synthesis of the Literature. BMC 14: 588.

8. Mickan S, Atherton H, Roberts NW, Heneghan C, Tilson JK (2014) Use of Handheld Computers in Clinical Practice; A Systematic Review. BMC Med Inf Dec Mak 14: 56.

9. Ventola C (2014) Mobile Devices and Apps for Health Care Professionals Uses and Benefits. P\&T 39(5): 356-364.

10. Webb K, Bullock A, Dimond R, Stacey M (2016) Can a Mobile App improve the Quality of Patient Care Provided by Trainee Doctors? Analysis of Trainee Reports. BMJ Open 6(9): 1-8.

11. Ayatollahi H, Sarabi FZP, Langarizadeh M (2015) Clinician's Knowledge and Perception of Telemedicine Technology. Perspective of Health Inform. Manage 12(Fall): 1c.

12. Cox A, Lucas G, Marcu A, Piano M, Grosvenor W, et al. (2017) Cancer Survivor's Experience with Telehealth: a Systematic Review and Thematic Synthesis. J Med Int Res 19(1): e11.

ISSN: 2574-1241

DOI: $10.26717 /$ BJSTR.2020.29.004806

Christie Kim. Biomed J Sci \& Tech Res

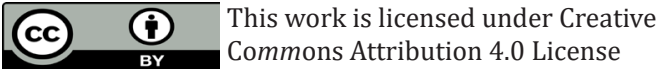

Submission Link: https://biomedres.us/submit-manuscript.php
13. Gordon HS, Solanki P, Bokhour BG, Gopal RK (2020) “I'm not Feeling like I'm part of the Conversation" Patient's Perspectives on Communicating in Clinical Video Telehealth Visits. Jour. Gen Int Med 35(6): 1751-1758.

14. Langarizadeh M, Moghbeli F, Aliabadi A (2017) Application of Ethics for Providing Telemedicine Services and Information Technology. Med Arch 71(5): 351-355

15. Nitarri G, Khuman R, Baldoni S, Pallotta G, Battineni G, et al. (2020) Telemedicine Practice: Review of the Current Ethical and Legal Challenges. Telemed J E Health.

16. Yip Y (2014) There's an App for that: the use of Mobile Medical Applications in Clinical Practice. Ontario Medical Review p. 1-4.

17. James M, Shaikh U, Steinhorn RH (2016) Addressing Health Disparities in Rural Communities using Telehealth. Ped Res 79(1-2): 169-176.

18. Fraser S, Mackean T, Grant J, Hunter K, Towers K, et al. (2017) Use of Telehealth for health care of Indigenous Peoples with Chronic Conditions. A Systematic Review. Rural and Remote Health 17(3): 4205.

$\begin{array}{ll}\text { BIOMEDICAL } & \text { Assets of Publishing with us } \\ \text { RESEARCHES } & \text { - Global archiving of articles } \\ & \text { - Immediate, unrestricted online access } \\ & \text { - Rigorous Peer Review Process } \\ \end{array}$

